\title{
Isolated Oropharyngeal Muscle Spasm in a Young Child post Extubation: A Case Report
}

\author{
Deepak Singla ${ }^{1}$, Abullais Raheeq Gowda², Vamshi Krishna², Shipra Tandon ${ }^{3}$

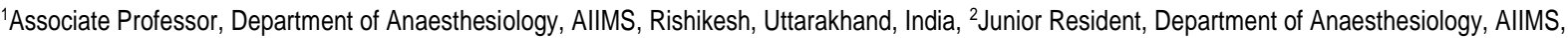 \\ Rishikesh, Uttarakhand, India, ${ }^{3}$ Senior Resident, Department of Anaesthesiology, AlIMS, Rishikesh, Uttarakhand, India.
}

\section{Abstract}

Agitation during emergence from anaesthesia is a documented phenomenon reported in children especially during immediate postoperative period; also Voluntary breath holding1and laryngospasm. This can lead to complications like postoperative distress, excessive crying, and anxiety. Here we report a case of postoperative respiratory distress in an 8 years old child of cerebral palsy developing severe oropharyngial spasm in the immediate postoperative period. Patient had no preoperative predisposing factors or intaraoperative events that could cause bronchospasm, laryngospasm, inadequate neuromuscular reversal or voluntary holding in the immediate postoperative period. Timely recognition and management of the condition prevented catastrophic complications like severe desaturation, hypoxic injury, negative pressure pulmonary edema or cardiac arrest.

Keywords: Isolated oropharyngeal muscle spasm, child, Voluntary breath holding, laryngospasm, brochospasm, cerebral palsy, desaturation.

Corresponding Author: Dr. Abullais Raheeq Gowda, Junior Resident, Department of Anaesthesiology, AIIMS, Rishikesh, Uttarakhand, India.

Email: abullaisg@gmail.com

Received: May 2020

Accepted: May 2020

\section{Introduction}

Children usually have a relatively higher degree of anxiety in the perioperative period as compared to adults. This may cause agitation during emergence from anaesthesia and lead to complications like postoperative distress, excessive crying, and anxiety. Voluntary breath-holding, ${ }^{[1]}$ laryngospasm are well-documented phenomena reported in such children, especially during the immediate postoperative period. ${ }^{[2]}$ Here we report a case of postoperative respiratory distress in a child associated with severe oropharyngeal spasm.

\section{Case Report}

8-year-old male child, case of cerebral palsy (spastic diplegia - lower limb) was posted for broomstick casting under general anaesthesia. The child had a birth history of delayed crying. There was no previous history of any breathlessness, excessive coughing, pneumonia, cyanotic episodes, seizures etc. All the routine investigations i.e. haemogram, urine routine and microscopy, were within normal limits. The patient was accepted under the American society of anesthesia (ASA) grade 1. On the night of surgery patient received dispersible lansoprazole tablet $15 \mathrm{mg}$ orally in 10 $\mathrm{ml}$ of apple juice. After confirming appropriate fasting inside $\mathrm{OT}$, routine monitors (pulse oximeter, non-invasive BP and
ECG) were attached. In the preoperative room patient's heart rate was $112 / \mathrm{min}$, blood pressure was $100 / 60 \mathrm{~mm} \mathrm{Hg}$ and saturation was $99 \%$ on room air. Oral midazolam was given to the child in a dose of $0.5 \mathrm{mg} / \mathrm{kg}$ with sips of water approximately 30 minutes before induction of anaesthesia.

The child was then taken inside operation theatre and after reattaching monitors, induced with inj. glycopyrrolate 0.004 $\mathrm{mg} / \mathrm{kg}$ intravenously (i.v.), inj. fentanyl $1.0 \mathrm{micro}-\mathrm{gm} / \mathrm{kg}$ i.v. and $8 \%$ sevoflurane. After confirming the adequacy of baf \& mask ventilation, inj. Vecuronium $0.1 \mathrm{mg} / \mathrm{kg}$ i.v. was given. The airway was secured with a cuffed endotracheal tube (ET) with an internal diameter of 5.0. The intraoperative period of 45 min was uneventful. During extubation, reversal was given with inj. neostigmine $1.5 \mathrm{mg}$ i.v. \&inj. glycopyrrolate $0.2 \mathrm{mg}$ i.v. it was decided to extubate the patient in a deep plane of anaesthesia. So, after confirming the adequacy of reversal (adequate respiratory efforts), eye-opening, limb movement, a proper oral suctioning was done and the ET was taken out.

After extubation patient was sedated, crying and agitated for some time. A few minutes later, the patient tightly clenched his jaw and started using accessory muscles of respiration, though crying was still present. He was not following verbal commands. The patient was given positive pressure ventilation via a mask, but it was not effective. Head tilt could not be done due to the rigidity of neck muscles. Even a suction catheter could not be passed beyond patient's nose. In the meantime, patient's saturation dropped from $99 \%$ to 
$85 \%$. Heart rate and blood pressure and temperature remained normal and there was no arrhythmia. Inj. Propofol $1.0 \mathrm{mg} / \mathrm{kg}$ i.v. was given to the patient. The patient responded to Propofol, and his jaw muscles became relaxed. It was possible to ventilate the patient. Patient's saturation gradually increased to $99 \%$ with $100 \%$ oxygen \& positive pressure ventilation by a mask. Gradually patient became conscious. Oxygen was started with a venturi mask at a fraction of 0.4 and the patient was comfortable with the saturation of $95 \%$. The patient was shifted to the postoperative care unit, where he was kept on oxygen by a venture mask. A chest $\mathrm{x}$-ray done in the postoperative room was clear. By evening patient was having a saturation of $98 \%$ on room air. So he was shifted to the ward. The rest of his hospital stay was also uneventful.

\section{Discussion}

Our child had had an acute episode of upper airway obstruction immediately after extubation. We diagnosed this condition to be a case of isolated oropharyngeal muscle spasm leading to complete airway obstruction. This is the first case report describing such a case in literature.

The various characters that we noted of this clinical condition were:

1. Age of children from 5 to 12 years.

2. No previous history of any respiratory or airway disease like asthma.

3. After surgery, the child is adequately meeting the criteria for reversal and good respiratory efforts until the endotracheal tube in situ.

4. Immediately after extubation, spasm of masseter muscles resulting in the clenching of the jaw along with a cessation of respiratory efforts.

5. At the time of spam, cry was present but decreased in amplitude

6. The inability of anaesthesiologist to open airway with head tilt chin lift maneuver.

7. Inability to pass suction catheter through mouth or nose.

The various differentials that can be considered in this condition are voluntary breath-holding by the child, presence of secretions or blood obstructing the airway, laryngospasm and bronchospasm, emergence agitation by sevoflurane or aggravation of pre-existing difficulty in swallowing due to cerebral palsy.

Voluntary breath-holding spells in infants have been described in a number of case reports for a long time. Few cases are reported in young children also 3 up to 4 years of age. Vurucus et al, ${ }^{[4]}$ described it to be related to delayed myelination of the brainstem. Our case was older than the children who may present with voluntary breath-holding. Also, the patient was not fully conscious. Even efforts to awake, the patient were futile. Also clinical features like clenching of jaw or spasm of upper airway muscles are not present in voluntary breath-holding. Lastly, as soon as the obstruction was relieved, good respiratory efforts returned. The presence of secretions or blood/blood clots could be another factor responsible for acute upper airway obstruction in the postoperative period. Though complete obstruction is rare, cases have been reported in the past with laryngospasm following reflux and aspiration. ${ }^{[5]}$ In our case, thorough oropharyngeal suctioning was done before extubation. The patient already received antacid prophylaxis. In addition, the chest on auscultation was clear after the airway obstruction was relieved without the need of suctioning. So secretion or blood as a cause of obstruction was unlikely.

Laryngospasm might also mimic the above-mentioned condition. Even the modalities we used for treatment could have been similar if this patient had an episode of laryngospasm. ${ }^{[6]}$ However it should be possible to open the mouth of the patient in case of a laryngospasm. The inability to open the mouth or pass a suction catheter through the oropharynx suggested it as the most likely site of obstruction. Also, characteristic stridor was absent in our case and during the initial part of obstruction, crying was present.

Emergence delirium is reported where induction and maintenance were done with volatile anesthetics like sevoflurane, with an incidence of $10 \%$ to $60 \% .^{[7]}$ Also, children who have high preoperative anxiety, hypoxia, airway compromises are, particularly at risk. ${ }^{[7]}$ However, emergence agitation presenting as airway obstruction is vary rare. Further various scores have been devised to quantify this problem of which PEAD score is most commonly used. Studies done in Asian children have shown that the PEAD score of $>10$ have a sensitivity of $0.98 .{ }^{[8]}$ However once the airway obstruction was relieved, the child was conscious, oriented, made eye contact and had no signs of restlessness or anxiety.

Problems in swallowing have been reported in children with cerebral palsy. ${ }^{\text {9] }}$ Such children have difficulty in swallowing due to bulbar muscle weakness and are at a higher risk of aspiration. However no such problem was there in our patient in the preoperative period. Patient was able to talk clearly and there was no history of the problem with feeding. Convulsion can also mimic the above presentation. However the patient was fully conscious once awake and no post-ictal phase was seen.

So after excluding all the known causes of upper airway obstruction in our patient we concluded that the obstruction might have resulted due to spasm of oropharyngeal muscles, resulting in a complete blockade and sudden desaturation. After a thorough search of literature, we could not find a similar presentation reported before. Management of our case was similar to any similar case. We immediately started positive pressure ventilation with a bag and mask. We were failing which we tried to relive the obstruction by airway maneuvers and oropharyngeal suctioning. When the above methods failed, we had to increase the depth of anaesthesia.

Fortunately, our patient recovered and did not suffer any complications. However if allowed to persist for some time this condition might result in negative pressure, pulmonary edema, severe desaturation or even arrest. So early recognition and prompt management of this condition are essential.

\section{References}

1. Goldman RD. Breath-holding spells in infants. Can Fam Physician. 2015;61(2):149-50. 
2. Visvanathan T, Kluger MT, Webb RK, Westhorpe RN. Crisis management during anaesthesia: laryngospasm. Qual Saf Health Care. 2005;14(3):3-6.

3. Evans OB. Breath-holding spells. Pediatr Ann. 1997;26(7):410-4.

4. Vurucu S, Karaoglu A, Paksu SM, Oz O, Yaman H, Gulgun M, et al. Breath-holding spells may be associated with the maturational delay in myelination of the brain stem. J Clin Neurophysiol. 2014;31(1):99101.

5. Bortolotti M. Laryngospasm and central reflex apnoea caused by aspiration of refluxed gastric content in adults. Gut 1989; 30: 233-238.
6. T Visvanathan T, Kluger MT, Webb RK, Westhorpe RN. Crisis management during anaesthesia: laryngospasm. Qual Saf Health Care $2005 ; 14: 3=5$.

7. Da Silva LM, Braz LG, Módolo NS. Emergence agitation in pediatric anesthesia: current features. J Pediatr (Rio J). 2008;84(2):107-113.

8. Bong CL, $\mathrm{Ng}$ AS. Evaluation of emergence delirium in Asian children using the Pediatric Anesthesia Emergence Delirium Scale.Paediatr Anaesth. 2009;19(6):593-600.

9. Prosser DP, Sharma N. Cerebral palsy and anaesthesia. Continuing education in anaesthesia critical care \& pain. 2010;10 (3): 72-76.

Copyright: () the author(s), 2020. It is an open-access article distributed under the terms of the Creative Commons Attribution License (CC BY 4.0), which permits authors to retain ownership of the copyright for their content, and allow anyone to download, reuse, reprint, modify, distribute and/or copy the content as long as the original authors and source are cited.

How to cite this article: Singla D, Gowda AR, Krishna V, Tandon S. Isolated Oropharyngeal Muscle Spasmin a Young Childpost Extubation: A Case Report. Acad. Anesthesiol. Int. 2020;5(1):162-164.

DOI: dx.doi.org/10.21276/aan.2020.5.1.33

Source of Support: Nil, Conflict of Interest: None declared. 